\title{
Analogies minus analogy test: measuring regularities in word embeddings
}

\author{
Louis Fournier ${ }^{1}$ \\ Emmanuel Dupoux ${ }^{12}$ \\ Ewan Dunbar 134 \\ ${ }^{1}$ Cognitive Machine Learning (ENS - CNRS - EHESS - INRIA - PSL Research University), France \\ ${ }^{2}$ Facebook A.I. Research, Paris, France \\ ${ }^{3}$ Laboratoire de Linguistique Formelle (CNRS - Paris Diderot - Sorbonne Paris Cite), France \\ ${ }^{4}$ University of Toronto, Toronto, Canada \\ louis.fournier@polytechnique.edu \\ ewan. dunbarautoronto.ca \\ emmanuel.dupoux@gmail.com
}

\begin{abstract}
Vector space models of words have long been claimed to capture linguistic regularities as simple vector translations, but problems have been raised with this claim. We decompose and empirically analyze the classic arithmetic word analogy test, to motivate two new metrics that address the issues with the standard test, and which distinguish between class-wise offset concentration (similar directions between pairs of words drawn from different broad classes, such as FranceLondon, China-Ottawa, ... ) and pairing consistency (the existence of a regular transformation between correctly-matched pairs such as France:Paris::China:Beijing). We show that, while the standard analogy test is flawed, several popular word embeddings do nevertheless encode linguistic regularities.
\end{abstract}

\section{Introduction}

Vector semantic models saw a surge in interest after embeddings trained under the word2 vec architecture (Mikolov et al., 2013a) were shown to encode linguistic regularities (Mikolov et al., 2013b). The demonstration relied on the arithmetic analogy test: relations such as king + woman - man $\approx$ queen were shown to hold for a variety of semantic and grammatical relations. Evaluation of word embeddings on analogy tests and training on related loss functions remains current. There is also continued interest in theoretically grounding the success of distributional embeddings on these tests (Allen and Hospedales, 2019; Ethayarajh et al., 2019).

There is, however, a substantial literature pointing to problems with word analogies (see Section 2 ), leading to the conclusion that word analogies are fragile in practice, and sometimes going so far as to imply that the positive results were erroneous. These critiques have been ambiguous as to whether the problem is that the embeddings in question do not really encode the relevant linguistic structure, or whether the issue is merely that the arithmetic analogy test as it is standardly defined, is flawed.

The current paper confirms that there are serious problems with the standard analogy test, as it confounds three different properties of word vectors while purporting to measure only one: class-wise offset concentration (similar directions between pairs of words drawn from different broad classes, such as France-London, China-Ottawa), withinpair similarity between test words (for example, the similarity between Paris and France), as well as the pairing consistency the test sets out to measure (the presence of a regular direction encoding relations such as capital-of: France-Paris, ChinaBeijing). We give an algebraic decomposition of the standard analogy test that explains previous negative results in terms of within-pair similarity. Using new measures, we show that, in practice, offset concentration, rather than true pairing consistency, may account for part of word embeddings' success. Nevertheless, we show that several standard word embeddings do show pairing consistency for the relations tested by the BATS analogy benchmark (Gladkova et al., 2016). ${ }^{1}$

\section{Related work}

The validity of the arithmetic analogy test has been questioned in several papers, starting with Levy and Goldberg (2014). We detail in Section 3 several major issues with the test as raised by Levy and Goldberg (2014), Linzen (2016), and Rogers et al. (2017). Finley et al. (2017), Newman-Griffis et al. (2017), Chen et al. (2017) and Schluter (2018) also raised concerns about the test and its assumptions. More recently, Nissim et al. (2020) argued against this test as an inadequate tool for studying bias in

\footnotetext{
${ }^{1}$ Code is available at github.com/bootphon/ measuring-regularities-in-word-embeddings.
} 
word embeddings. Rogers (2019) observes that many of the issues have been ignored.

Some works have proposed other measures of linguistic relations in word embeddings. Levy and Goldberg (2014), Vylomova et al. (2016), and Rogers et al. (2017) all examined the similarity of vector offsets in a more direct way than the standard analogy test (see Section 3 below). Drozd et al. (2016) proposed a method based on predicting the class of the unknown word, and Bouraoui et al. (2018) relaxed the assumptions by allowing probabilistic models to predict the relations.

We claim that these works still do not provide a satisfactory measure of how well word vector offsets encode linguistic relations. Without such a measure, it is impossible to assess whether the original conclusions are correct. We develop this argument, and then develop a new measure, below.

\section{The arithmetic analogy test}

Mikolov et al. (2013b) proposed to measure the presence of linguistic relations in the structure of word embeddings using vector arithmetic. For two pairs of words representing the same linguistic relation, $\left(a, a^{*}\right)$ and $\left(b, b^{*}\right)$, the test assesses whether:

$$
b+\left(a^{*}-a\right) \approx b^{*}
$$

In practice, the test assesses whether $b^{*}$ is the nearest neighbour to $b+a^{*}-a$, using the cosine similarity (cosine of the angle between $x$ and $y$ ):

$$
\operatorname{sim}(x, y)=\frac{x \cdot y}{\|x\|\|y\|}
$$

The assumption is that, if the offset $o_{a}=a^{*}-a$ is parallel to the offset $o_{b}=b^{*}-b$, then this common offset direction encodes the same linguistic relation. For example, if Paris minus France goes in the same direction as Beijing minus China, and as other capital-country pairs, the common offset direction can be seen as encoding the relation capital-of.

Thus, the idea behind the arithmetic analogy test is that offsets corresponding to the same linguistic relation, if they are the same or roughly the same, should be interchangeable. Therefore, France $+($ Beijing - China $)$ should be the same as France + (Paris - France) (modulo the vector magnitude, if we are hypothesizing that it is the direction, and not the precise normed vector, that encodes the relation capital-of: hence the use of the cosine similarity).
The criticisms of Mikolov et al. (2013b) and the research it inspired have often been characterized as problems with "word analogies." This is ambiguous: it does not distinguish between problems with the method of using the arithmetic test just described for testing the presence of linguistic regularities, and the veracity of the conclusion that there are linguistic regularities coded in word embeddings as vector offsets. In order to resolve this ambiguity, one would need to know for sure, using some better measure, whether linguistic regularities are coded. We propose such a measure beginning in Section 4. We first analyze the key problems that have been previously raised.

\subsection{Within-pair similarity}

The analogy will be scored correct if $b^{*}$ is the $\arg \max _{x} \operatorname{sim}\left(b+o_{a}, x\right)$. Levy and Goldberg (2014) call this objective 3Cos ADD. Let us call $\operatorname{sim}\left(b+o_{a}, b^{*}\right)$ the "analogy score." Putting aside norms, the analogy score can be decomposed as:

$$
\begin{aligned}
\operatorname{sim}\left(b+o_{a}, b^{*}\right) & \propto b \cdot b^{*}+o_{a} \cdot b^{*} \\
& \propto b \cdot b^{*}+o_{a} \cdot o_{b}+o_{a} \cdot b
\end{aligned}
$$

The first term, the dot product of $b$ and $b^{*}$, is proportional to the within-pair similarity, $\operatorname{sim}\left(b, b^{*}\right)$. The second term is proportional to the similarity between the offsets, which would appear at first glance, to be the term of principal interest in assessing whether a linguistic relation is consistently coded by the offsets. The third term is proportional to the similarity between the start word $b$ and the offset $o_{a}$, and does not depend on $b^{*}$.

We develop an analysis of the offset similarity in sections 4-5, deriving from it the offset concentration and pairing consistency properties we propose to measure. Previous work suggested that offset similarity is not very high in practice, compared to within-pair similarity. Levy and Goldberg (2014) showed that evaluating on the basis of similarity between offsets, using PAIRDIRECTION to determine the nearest neighbours, leads to failure on analogy tests that would otherwise succeed using the 3COSADD objective. Rogers et al. (2017) showed that within-pair similarity is correlated with performance on the standard arithmetic analogy test. Clearly, measuring whether Paris is similar to France is not the same as measuring whether the relation capital-of is present in the word embedding. As Levy and Goldberg point out, within-pair similarity is not irrelevant to the 
question-if the idea is that there is a consistent dimension capital-of which should constitute the principal difference between Paris and France, then the two words should otherwise be similar. But a test dominated by within-pair similarity can lead to spurious results, since pairs of words may be similar without capturing the relation in question.

Using the Bigger Analogy Test Set (BATS) (Gladkova et al., 2016) and the pre-trained Google News skip-gram embeddings of Mikolov et al. (2013a), we examine the role of the three terms of (3). BATS consists of forty relations, each composed of fifty word pairs, grouped into four broad types: inflectional morphology, derivational morphology, encyclopedic semantics (like capitals and animal sounds), and lexicographic semantics (like synonyms and antonyms). When multiple pairs exist for the same input word, we only keep the first. For each of the forty relations, we take the mean for each term over all $a: a^{*}:: b: b^{*}$ tuples.

Complementing previous analyses, we display means for these three terms in Figure 1, confirming that within-pair similarity is indeed positive and large compared to the other terms. Notably, offset similarities vary between relations, but are almost always smaller. However, they are always positive.

\subsection{Honest analogies: predicting input words}

Linzen (2016) observes that the arithmetic analogy test, as practised, excludes $a, a^{*}$, and $b$ from the arg max-otherwise, one of these input words tends (incorrectly) to be the response-most of the time, $b$. Rogers et al. (2017) and Schluter (2018) imply that part of the issue may be that $\left\|o_{a}\right\|$ is small. This cannot be why: the offsets have similar magnitudes, so if $\left\|o_{b}\right\|$ is long enough to bring $b$ to $b^{*}$, then $\left\|o_{a}\right\|$ should be too. Importantly, if we compute the decomposition in (3), but suppose that $b^{*}$ is equal to $b+o_{a}\left(o_{a}=o_{b}\right.$ : the analogy score is thus always 1 ), we observe empirically that the within-pair similarity- and offset similaritydriven terms have similar values (Figure 8 in the appendix). This means that $\left\|o_{a}\right\|$ is similar to $\|b\|$. The weakness of the offset-similarity term in the score is not driven by the offsets being small.

Define $\Delta_{\text {sim }}$ as the analogy score minus the similarity of $b+o_{a}$ to the start word $b$, which must be negative in order for the "honest" analogy test to return $b$. We observe that $\Delta_{\text {sim }}$ is equal to:

$$
\Delta_{\text {sim }}=\frac{b+o_{a}}{\left\|b+o_{a}\right\|} \cdot\left(\frac{b^{*}}{\left\|b^{*}\right\|}-\frac{b}{\|b\|}\right)
$$

By replacing $b^{*}$ by $b+o_{a}$, we can expand the score, which is then proportional to:

$$
\frac{\|b\|-\left\|b^{*}\right\|}{\|b\|} \cdot\left(b+o_{a}\right) \cdot b+o_{a} \cdot o_{b}+b \cdot o_{b}
$$

This decomposes $\Delta_{\text {sim }}$ into three terms. The first term is proportional to the difference between the norms of $b$ and $b^{*}$, which we have empirically observed to be small, and which will be null for normalized embeddings. The second term is proportional to the offset similarity, and the third term is proportional to the similarity between $b$ and $o_{b}$. For normalized word vectors, the third term is equal to $\operatorname{sim}\left(b, b^{*}\right)-1$, which is the negative cosine distance between $b$ and $b^{*}$. Since $\|b\|$ and $\left\|b^{*}\right\|$ tend to be similar, we find that this is a good approximation even for non-normalized vectors. $\Delta_{\text {sim }}$ is thus negative, roughly, whenever the offset similarity is less than the cosine distance between $b$ and $b^{*}$. Intuitively, the similarity of the offsets must compensate for the difference between $b^{*}$ and $b$.

In Figure 2, we plot means for the three terms from (5). We observe that the first term is indeed small and can be ignored. Importantly, we observe that the offset similarity term is smaller in magnitude than the negative $b-b^{*}$ distance term).

Linzen's observation that an input word is typically predicted under the 3COSADD objectiveand not an unrelated vocabulary word-implies that not only is $\Delta_{\text {sim }}$ negative, but that the equivalent value is negative for all words in the vocabulary. This in turn would imply (for normalized embeddings) that the similarity between $o_{a}$ and the offset of $b$ with respect to any other word is smaller than the distance between $b$ and that word. Far from staying in the neighbourhood of $b, o_{a}$ moves $b$ in a direction far from every word in the vocabulary.

Our analysis of these known problems with word analogies details the central problem: the pairs of offsets tested are not similar enough to support the kind of arithmetic demanded by the 3COSADD objective. An illustration of the dominance of withinpair similarity is given in Figure 3. Note that we present this figure only to illustrate the dominance of within-pair similarity. Two-dimensional projections are misleading: the vectors in Figure 3 do not have the property, discussed above, that the offsets are of length similar to the word vectors themselves-this is difficult to reconcile with high within-pair similarity in two dimensions.

None of this, however, implies that word embed- 


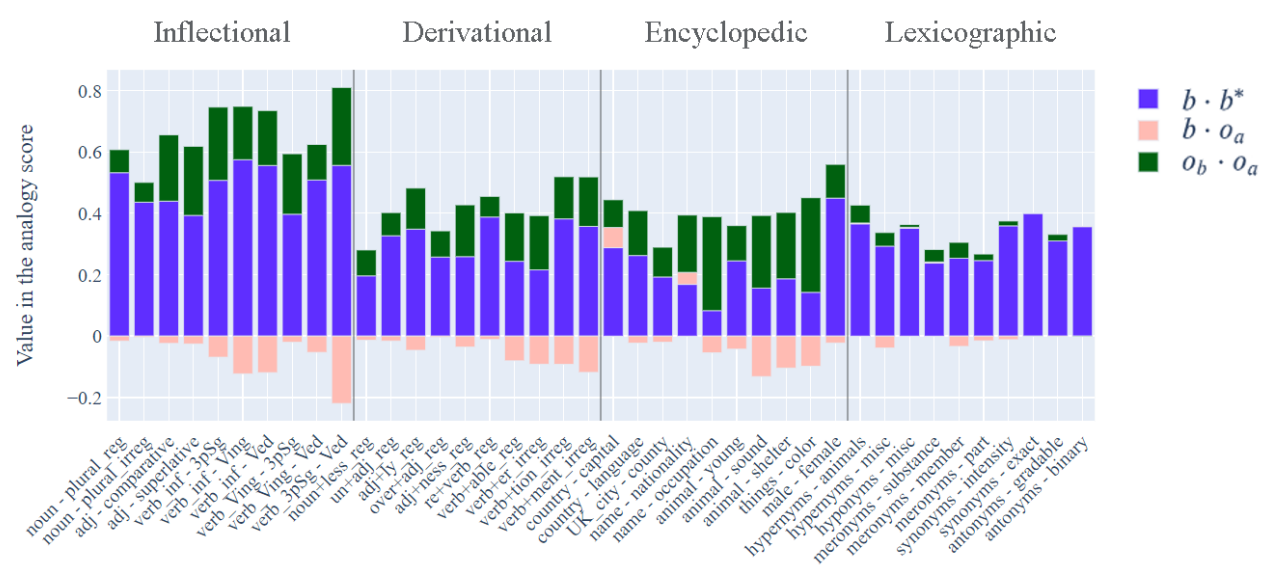

Figure 1: Decomposition of the analogy score in three terms, with only two depending on the word to predict. The largest component is $b \cdot b^{*}$, proportional to within-pair similarity. $b \cdot o_{a}$ is proportional to the similarity of the offset to the start word $b . o_{b} \cdot o_{a}$ is proportional to the similarity between offsets. All terms are divided by the same overall normalization term (not indicated in the legend).

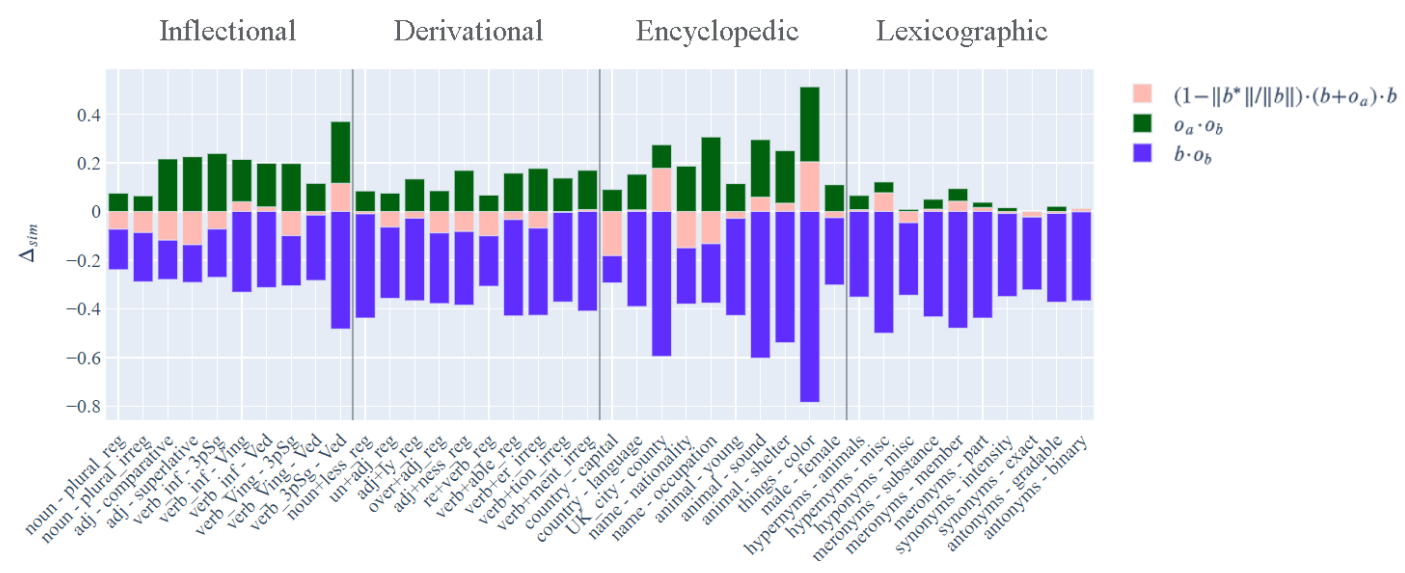

Figure 2: Decomposition of $\Delta_{\text {sim }}$, the difference between the similarity of the analogy to $b^{*}$ and to $b$. The term proportional to the dissimilarity of $b$ to $b^{*}$ is greater than the one proportional to the offset similarity, which means the common direction of the offset is not enough to bring the analogy closer to $b^{*}$ than to $b$.

dings do not show linguistic regularities. It simply implies that the standard arithmetic analogy test does not measure these regularities correctly, and should not be used. While it may be true that the quantity of interest, the similarity between offsets, is generally insufficient to dominate the standard analogy test, that does not mean it is too small to be considered an encoding of a linguistic regularity. How similar is similar enough? In the following section, we propose an answer to this question.

\section{Measuring Regularities}

The similarity between offsets, $\operatorname{sim}\left(o_{a}, o_{b}\right)$, is the quantity of primary interest to assess whether linguistic relations are represented as consistent directions. We seek a measure of whether they are. As noted, Levy and Goldberg (2014) showed that $\arg \max _{x} \operatorname{sim}\left(a^{*}-a, x-b\right)$ does not tend to find the right answer $\left(b^{*}\right)$. However, the fact that $b^{*}$ does not maximize offset similarity does not answer the question of what a meaningful level of offset similarity is. Rogers et al. (2017) analyzed examples of correct and false analogies and showed them to have comparable offset similarities. This makes sense: a baseline level of offset similarity can be found by looking at unrelated pairs. However, that paper examined only one isolated example.

We propose an offset concentration score (OCS) measuring the offset similarity among pairs belonging to the same relation and a pairing consistency score (PCS) measuring the distinctness of the similarities from a baseline. We propose PCS as the correct measure of linguistic regularity, with OCS a source of supplementary information. 

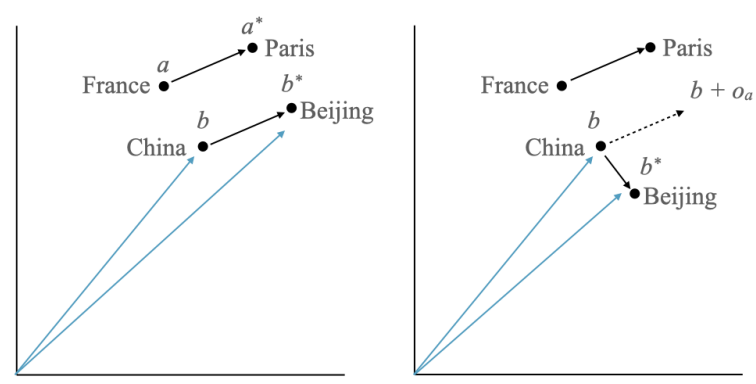

Figure 3: Schematic illustration of an analogy underlining the role of within-pair similarity in the arithmetic analogy test. At left, China + (Paris - France) predicts Beijing in part for the "right" reason (offsets are parallel). At right, the offsets are not parallel, but $\mathrm{Bei}$ jing is likely to be predicted, since its cosine similarity to China is high. The problem is worse than the 2D schematic would suggests, since it can occur even when the word vectors are normalized, and when the offsets are quite long. China will be predicted if it is not excluded, since it is also in the neighbourhood.

Perfectly parallel offsets would imply that France and Paris differ on the same dimension as China and Beijing - and on no other dimensionand similarly for the other offsets of the same kind (Canada-Ottawa,...). Perfect parallelism is not necessary for $\operatorname{sim}\left(o_{a}, o_{b}\right)$ to contribute to success on the analogy test. By offset concentration, we mean the degree to which, for an entire set of word pairs, the offsets are parallel to one another (thus, concentrated in a single direction).

Once we take into account that offsets need not be perfectly parallel, we must bear in mind that positive cosine similarity between offsets does not imply that the embedding space captures linguistic relations. For example, recalling Schluter (2018), training embeddings to capture distributional regularities may group words into linguistic classeswith, for example, country names such as France, China..., occupying a region of the space distinct from that of Paris, Beijijng..., due to distributional commonalities. This by itself could yield offset concentration: offsets would all come from a similar origin, and go to a similar destination. However, $\operatorname{sim}$ (France-Paris, China-Beijing) would not necessarily be larger than $\operatorname{sim}$ (France-Beijing, ChinaParis). We illustrate this issue in Figure 4.

In such an example, we might be able to assert that the class of capital city names is distinct from the class of country names. But asserting that the relation capital-of is captured is a stronger property. It would require that moving from the France

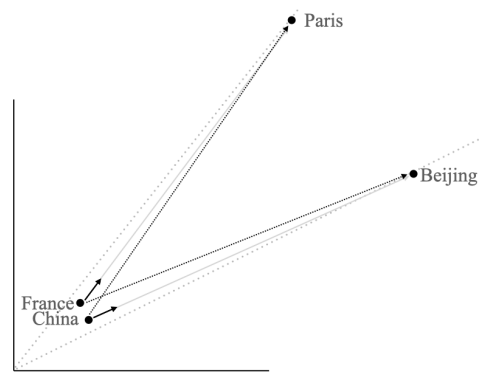

Figure 4: Schematic illustration of an analogy underlining the pitfalls of offset concentration. At left, Paris - France appears nearly orthogonal to Beijing - China. At right, however, the offsets have much greater cosine similarity, simply because Paris and Beijing are far away. The similarity between Paris - China and Beijing - France (dotted lines) is almost as high. Unlike what might be suggested by the 2D schema, this can arise easily even for normalized word vectors.

vector in the direction of the common capital-of vector lead to Paris and not to Beijing, nor to any other capital city - or, at least, that it passes closer to Paris. We call this property pairing consistency.

\subsection{Offset concentration}

Figure 5 shows a histogram of the pairwise similarities between all pairs of BATS analogy offsets for the pre-trained word2vec embeddings used above, grouped by broad BATS relation type. As above, these similarities are small but generally positive. We propose an offset concentration score:

$$
\mathrm{OCS}=\frac{1}{N(N-1)} \sum_{i} \sum_{j \neq i} o_{i} \cdot o_{j}
$$

which is the mean similarity between normalized offsets within a single relation, where the normalized offset vector $o_{i}$ of a given word pair is $o_{i}=\frac{a_{i}^{*}-a_{i}}{\left\|a_{i}^{*}-a_{i}\right\|}$. We discuss in the appendix the link between the OCS and the mean direction of the offsets.

\subsection{Pairing consistency}

To measure the pairing consistency, we compare the direction of the offsets for a given set of word pairs against comparable shuffled offsets from the same BATS relation. Shuffled offsets are generated by holding the start words constant but permuting the end words (avoiding true pairs). We expect true offsets to be more parallel than shuffled offsets.

We sample sets of shuffled offsets for each relation. For each set, as for the true offsets, we 


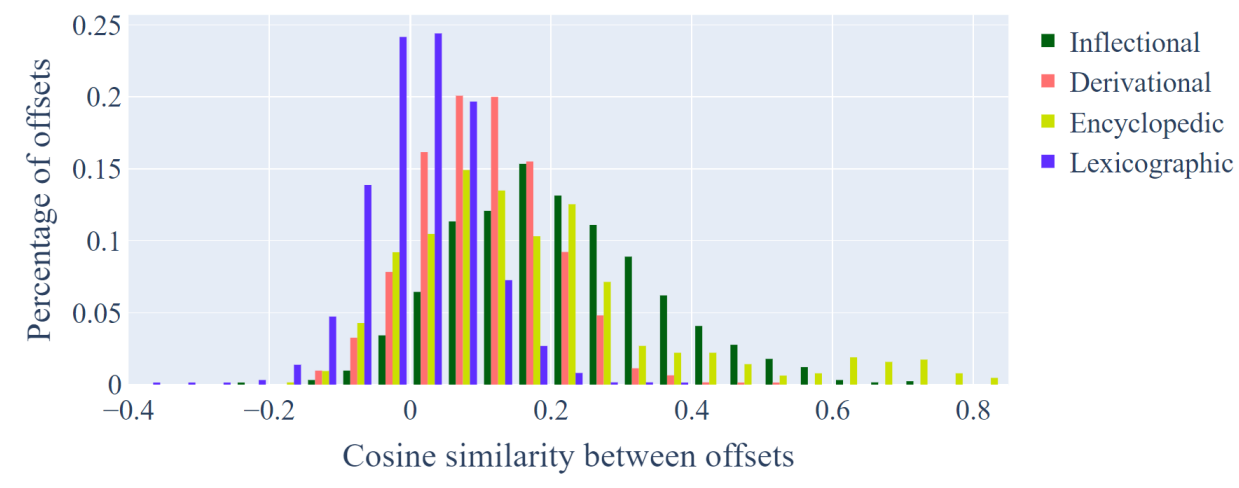

Figure 5: Distribution of the cosine similarities between vector offsets for word2vec embeddings, grouped by broad relation type. The Offset Concentration Score is the mean offset similarity for all pairs within a single relation (e.g., country-capital).

compute all pairwise similarities between offsets. Rather than averaging similarities as in the OCS, we calculate the separation between distributionsthe true offset similarities versus each set of shuffled offset similarities. We empirically construct the receiver operating characteristic curve for linearly separating true versus shuffled similarities (i.e., setting a criterion on the similarity dimension above which offsets are true offsets and below which offsets are shuffled offsets). We calculate the area under the curve (AUC). The AUC will be 1 for perfectly separable distributions, 0.5 for indistinguishable distributions, and below 0.5 when true offsets are systematically less parallel than the baseline. We average AUC over all shuffled sets.

For any set of offsets $O$, we refer to the set of its offset similarities as $\operatorname{sim}(O)=\left\{o_{i} \cdot o_{j}, \forall\left(o_{i}, o_{j}\right) \in\right.$ $\left.O^{2}, i<j\right\}$. For $S_{s}$ a list of $N_{s}$ sets of shuffled offsets $O^{\prime}$ (here $N_{s}=50$ ), we define the following pairing consistency score, where $O$ are the true offsets:

$$
\mathrm{PCS}=\frac{1}{N_{s}} \sum_{O^{\prime} \in S_{s}} \operatorname{AUC}\left(\operatorname{sim}(O), \operatorname{sim}\left(O^{\prime}\right)\right)
$$

\section{Exploration of word2vec}

We perform a series of experiments to assess the presence of linguistic regularities in the pre-trained word2vec embeddings used above, and to show the behavior of our new measures.

\subsection{Random baselines}

In addition to the real BATS test, we apply our measures to artificial analogy sets, which we construct to show no pairing consistency. We seek to justify the use of pairing consistency, rather than directly using offset concentration, by demonstrating that offset concentrations can remain non-zero even for arbitrarily associated pairs. For each BATS relation, we construct ten of each of the following types of permuted relations. We calculate the mean OCS and the mean PCS over the ten instances. We expect the mean PCS to be 0.5 in each case.

First, we construct sets of word pairs derived from the BATS by permutation within-category: for each category, the end words are randomly reassigned to start words. In other words, we construct categories for which shuffling should have no effect (they are already shuffled). Comparing the OCS to that of the real BATS also allows us to measure the effect of shuffling on the OCS.

If permuted word pairs can show positive offset concentration simply because the start words and/or the end words are found in a coherent region of the embedding space, then positive OCS should be found in word pairs drawn from mismatched categories. For each pair of start/end word categories among the BATS relations (for example, the noun-plural_reg relation maps the start category singular nouns to the end category regular plural nouns), we randomly re-assign the end category (yielding, for example, singular nouns-past tense verbs). We then randomly construct fifty start/end pairs. We compare mismatched categories from within versus across broad BATS category types (inflectional morphology, derivational morphology, encyclopedic semantics, lexicographic semantics).

Finally, to assess the impact of the geometric coherence of words within categories on the OCS, we construct pairs without respect for category: we compare word pairs with random start words, random end words, and random start and end words, with ten different random word sets. 


\subsection{Offset concentration}

Table 1 shows the OCS and PCS scores on real and randomized BATS relations using word2vec. The OCS scores for the real BATS sets are not close to one (as expected, given the results above).

The fully random set has an OCS of zero, confirming that offset concentration is unlikely to occur by chance, at least for these word vectors. The other random analogy sets all show non-zero OCS. Some random sets have even higher OCS scores than the real BATS sets. Thus, as predicted, offset concentration can exist even when linguistic relations are, by construction, not coded in any systematic way. Even the random-start and random-end pairs show non-zero $\mathrm{OCS},{ }^{2}$ indicating that geometric coherence within one category is sufficient to give some degree of parallelism to the offsets. Surprisingly, the OCS is systematically lower for the permuted within-category baseline than for the mismatched-category baselines. Mismatched categories may be further apart than matched categories, reducing the angles between offsets overall.

These results have important consequences for how we measure meaningful regularities in vector embeddings. Not only is the arithmetic analogy test not fit to detect the presence of similar directions among related pairs, even if it were, similar directions alone are not sufficient to deduce that linguistic regularities are coded. The test can be dominated by spurious within-pair similarity and by spurious offset concentration-which can arise even when matching against random words.

\subsection{Pairing consistency}

The real BATS sets show PCS above chance, contrary to all random analogy sets, which show PCS of 0.5 , as expected. ${ }^{3}$ The PCS scores are low for the semantic (encyclopedic, lexicographic) relations.

In Section 3 above, we remarked that within-pair similarity is not completely irrelevant to analogies, as a higher within-pair similarity amplifies the component along the common direction. Here, the relations which show greater within-pair similarity (Figure 1) also show higher PCS.

\footnotetext{
${ }^{2}$ We observe a systematic difference between the random start and random end results, which seems to indicate a bias in the BATS analogy sets, with the end words being more susceptible to forming clusters.

${ }^{3}$ As noted, these scores are the mean over ten replications of the random sets. Even where the mean is not precisely 0.5 , it is always within one half-interquartile-range of 0.5.
}

\section{Comparing word embeddings}

We evaluate the pairing consistency on the BATS test for popular word embeddings. We include Glove (Pennington et al., 2014), a purely distributional model similar to word2vec; two embeddings making use of external semantic knowledge: dict2vec (Tissier et al., 2017) and ConceptNet Numberbatch (Speer et al., 2017); and the static token embeddings of BERT (Devlin et al., 2019) and GPT-2 (Radford et al., 2019).

The left subfigure in Figure 6 presents the standard 3COSADD analogy test accuracy (input words excluded). Within broad relation types, we see similar results across embeddings, with a few exceptions (the static GPT-2 embeddings are poor throughout, but much better than the others on derivational morphology). Consistent with previous results using the arithmetic analogy test, derivational morphology and encyclopedic and lexical semantic relations obtain lower scores than inflectional morphology (Gladkova et al., 2016; Rogers et al., 2017).

The PCS scores are presented at right in Figure 6. The general picture is similar: there are systematic differences between relation types, and inflectional morphology tends to be well coded. However, contrary to the conclusions of the standard analogy test, the PCS reveals that Numberbatch codes linguistic relations better than the other embeddings, which is more consistent with the evaluations presented in Speer et al. (2017). The PCS also shows that derivational morphology occupies an intermediate position between inflectional morphology and semantics: word2vec, Glove, and Numberbatch in fact code derivational morphology better, in the PCS sense, than encyclopedic semantic relations. The elevated analogy test performance of some embeddings on encyclopedic relations is likely an artefact of the kind of category-level geometric coherence discussed in Section 5. As we show in the appendix in Table 2 , encyclopedic relations show higher OCS overall than derivational morphology relations, in spite of lower PCS.

\section{Contributions}

We have made new arguments against the use of the standard arithmetic analogy test in the evaluation of word embeddings. We show in detail how regularities in vector offsets, which are the object of primary interest for the analogy test, are, on the one hand, washed out by within-pair similarity, and, 


\begin{tabular}{|c|c|c|c|c|c|c|c|c|}
\hline \multirow[b]{2}{*}{ Analogy set } & \multicolumn{2}{|c|}{ Inflectional } & \multicolumn{2}{|c|}{ Derivational } & \multicolumn{2}{|c|}{ Encyclopedic } & \multicolumn{2}{|c|}{ Lexicographic } \\
\hline & OCS & PCS & OCS & PCS & OCS & PCS & OCS & PCS \\
\hline Real BATS & .295 & .851 & .156 & .679 & .198 & .559 & .031 & .539 \\
\hline Permuted within-category & .111 & .500 & .088 & .500 & .170 & .500 & .015 & .500 \\
\hline Mismatched category (within type) & .147 & .501 & .120 & .500 & .260 & .501 & .093 & .500 \\
\hline Mismatched category (across type) & .175 & .500 & .173 & .500 & .223 & .500 & .134 & .500 \\
\hline Random start & .090 & .500 & .075 & .500 & .204 & .499 & .069 & .500 \\
\hline Random end & .063 & .500 & .060 & .500 & .137 & .501 & .059 & .499 \\
\hline Random start and end & .000 & .500 & & & & & & \\
\hline
\end{tabular}

Table 1: Offset Concentration Scores and Pairing Consistency Scores for the real and random baseline analogy sets (higher is better; 0.5 is chance level for PCS). Random baseline scores are averaged across ten permutations. All scores are then averaged across BATS relations. Mismatched categories (across broad BATS relation type) are grouped along the start type. Random start and end is not assigned to a BATS type, as all the words are drawn randomly. Bold indicates the highest OCS score in a given column: we see that OCS is not always highest for real analogies. Random baseline PCS scores are always within a half-IQR of 0.5 .
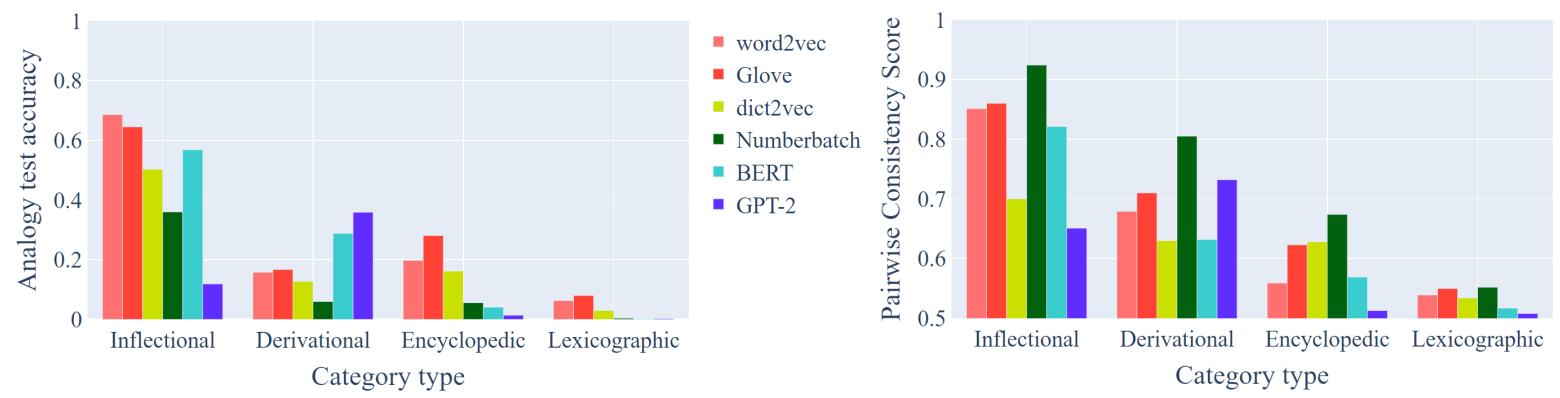

Figure 6: Left: accuracy on the standard arithmetic analogy test, across embeddings, averaged across BATS relations (within the four broad types). Right: the same figure for the Pairing Consistency Score, starting at .5 (chance-level).

on the other, insufficient to establish the existence of linguistic regularities. We explain the previously observed phenomenon that arithmetic analogy tests consistently predict the input words.

We propose a new measure of the presence of linguistic relations in word embeddings, the pairing consistency score, which measures the degree to which offsets between related pairs are parallel above chance, and the offset concentration score, a complementary measure of the absolute degree of parallelism. We show that a variety of word vectors really do capture linguistic regularities, in spite of distortions introduced by the arithmetic analogy test. We show that these distortions lead to spurious conclusions when comparing performance. Arithmetic analogy tests and loss functions based on them are deprecated and should be replaced by direct evaluation of pairing consistency.

\section{Acknowledgments}

This work was funded in part by the European Research Council (ERC-2011-AdG-295810 BOOTPHON), the Agence Nationale pour la
Recherche (ANR-17-EURE-0017 Frontcog, ANR17-CE28-0009 GEOMPHON, ANR-10-IDEX0001-02 PSL*, ANR-19-P3IA-0001 PRAIRIE 3IA Institute, ANR-18-IDEX-0001 U de Paris, ANR10-LABX-0083 EFL) and grants from CIFAR (Learning in Machines and Brains), Facebook AI Research (Research Grant), Google (Faculty Research Award), Microsoft Research (Azure Credits and Grant), and Amazon Web Service (AWS Research Credits).

\section{References}

Carl Allen and Timothy Hospedales. 2019. Analogies Explained: Towards Understanding Word Embeddings. In Proceedings of the 36th International Conference on Machine Learning, volume 97 of Proceedings of Machine Learning Research, pages 223 231, Long Beach, California, USA. PMLR.

Zied Bouraoui, Shoaib Jameel, and Steven Schockaert. 2018. Relation Induction in Word Embeddings Revisited. In Proceedings of the 27th International Conference on Computational Linguistics, pages 1627-1637, Santa Fe, New Mexico, USA. Association for Computational Linguistics. 
Dawn Chen, Joshua C. Peterson, and Thomas L. Griffiths. 2017. Evaluating vector-space models of analogy. CoRR, abs/1705.04416.

Jacob Devlin, Ming-Wei Chang, Kenton Lee, and Kristina Toutanova. 2019. BERT: Pre-training of Deep Bidirectional Transformers for Language Understanding. In Proceedings of the 2019 Conference of the North American Chapter of the Association for Computational Linguistics: Human Language Technologies, Volume 1 (Long and Short Papers), pages 4171-4186, Minneapolis, Minnesota. Association for Computational Linguistics.

Aleksandr Drozd, Anna Gladkova, and Satoshi Matsuoka. 2016. Word Embeddings, Analogies, and Machine Learning: Beyond King - Man + Woman = Queen. In Proceedings of COLING 2016, the 26th International Conference on Computational Linguistics: Technical Papers, pages 3519-3530, Osaka, Japan, December 11-17.

Kawin Ethayarajh, David Duvenaud, and Graeme Hirst. 2019. Towards Understanding Linear Word Analogies. In Proceedings of the 57th Annual Meeting of the Association for Computational Linguistics, pages 3253-3262, Florence, Italy. Association for Computational Linguistics.

Gregory Finley, Stephanie Farmer, and Serguei Pakhomov. 2017. What Analogies Reveal about Word Vectors and their Compositionality. In Proceedings of the 6th Joint Conference on Lexical and Computational Semantics (*SEM 2017), pages 1-11, Vancouver, Canada. Association for Computational Linguistics.

Anna Gladkova, Aleksandr Drozd, and Satoshi Matsuoka. 2016. Analogy-Based Detection of Morphological and Semantic Relations with Word Embeddings: What Works and What Doesn't. In Proceedings of the NAACL-HLT SRW, pages 47-54, San Diego, California, June 12-17, 2016. ACL.

Omer Levy and Yoav Goldberg. 2014. Linguistic Regularities in Sparse and Explicit Word Representations. In Proceedings of the Eighteenth Conference on Computational Natural Language Learning, pages 171-180, Ann Arbor, Michigan. Association for Computational Linguistics.

Tal Linzen. 2016. Issues in Evaluating Semantic Spaces Using Word Analogies. In Proceedings of the First Workshop on Evaluating Vector Space Representations for NLP. Association for Computational Linguistics.

Tomas Mikolov, Kai Chen, Greg Corrado, and Jeffrey Dean. 2013a. Efficient Estimation of Word Representations in Vector Space. In Proceedings of International Conference on Learning Representations (ICLR).

Tomas Mikolov, Wen-tau Yih, and Geoffrey Zweig. 2013b. Linguistic Regularities in Continuous Space
Word Representations. In Proceedings of NAACLHLT 2013, pages 746-751, Atlanta, Georgia, 9-14 June 2013.

Denis Newman-Griffis, Albert Lai, and Eric FoslerLussier. 2017. Insights into Analogy Completion from the Biomedical Domain. In BioNLP 2017, pages 19-28, Vancouver, Canada,. Association for Computational Linguistics.

Malvina Nissim, Rik van Noord, and Rob van der Goot. 2020. Fair Is Better than Sensational: Man Is to Doctor as Woman Is to Doctor. Computational Linguistics, 46(2):487-497.

Jeffrey Pennington, Richard Socher, and Christopher Manning. 2014. GloVe: Global Vectors for Word Representation. In Proceedings of the 2014 Conference on Empirical Methods in Natural Language Processing (EMNLP), pages 1532-1543, Doha, Qatar. Association for Computational Linguistics.

Alec Radford, Jeffrey Wu, Rewon Child, David Luan, Dario Amodei, and Ilya Sutskever. 2019. Language Models are Unsupervised Multitask Learners. OpenAI Blog, 1(8):9.

Anna Rogers. 2019. On word analogies and negative results in NLP. Hacking Semantics.

Anna Rogers, Aleksandr Drozd, and Bofang Li. 2017. The (Too Many) Problems of Analogical Reasoning with Word Vectors. In Proceedings of the 6th Joint Conference on Lexical and Computational Semantics (* SEM 2017), pages 135-148.

Natalie Schluter. 2018. The Word Analogy Testing Caveat. In Proceedings of the 2018 Conference of the North American Chapter of the Association for Computational Linguistics: Human Language Technologies, Volume 2 (Short Papers), pages 242-246, New Orleans, Louisiana. Association for Computational Linguistics.

Robyn Speer, Joshua Chin, and Catherine Havasi. 2017. ConceptNet 5.5: An Open Multilingual Graph of General Knowledge. In Proceedings of the ThirtyFirst AAAI Conference on Artificial Intelligence, AAAI'17, page 4444-4451. AAAI Press.

Julien Tissier, Christophe Gravier, and Amaury Habrard. 2017. Dict2vec : Learning Word Embeddings using Lexical Dictionaries. In Proceedings of the 2017 Conference on Empirical Methods in Natural Language Processing, pages 254-263, Copenhagen, Denmark. Association for Computational Linguistics.

Ekaterina Vylomova, Laura Rimell, Trevor Cohn, and Timothy Baldwin. 2016. Take and Took, Gaggle and Goose, Book and Read: Evaluating the Utility of Vector Differences for Lexical Relation Learning. In Proceedings of the 54th Annual Meeting of the Association for Computational Linguistics (Volume 1: Long Papers), pages 1671-1682, Berlin, Germany. Association for Computational Linguistics. 


\section{A The offsets' mean direction}

For an offsets set, the OCS measures how concentrated the hypercone of the offsets is. The center of this hypercone is the average normalized offset $d$, the unit vector defined by

$$
d \propto \sum_{i} \frac{a_{i}^{*}-a_{i}}{\left\|a_{i}^{*}-a_{i}\right\|}
$$

This vector has different interesting properties. First, we can note that it is the unit vector maximising the sum of the similarities of the offsets with it. With the normalized offsets $o_{i}=\frac{a_{i}^{*}-a_{i}}{\left\|a_{i}^{*}-a_{i}\right\|}$,

$$
d=\underset{u,\|u\|=1}{\arg \max } \sum_{i} o_{i} \cdot u
$$

Furthermore, let's define MSM, the mean similarity of $d$ to the offsets. We can first prove that the MSM is equal to the norm of $d$ before normalization:

$$
\begin{aligned}
\operatorname{MSM} & =\frac{1}{N} \sum_{j} \operatorname{sim}\left(\frac{\frac{1}{N} \sum_{i} o_{i}}{\left\|\frac{1}{N} \sum_{i} o_{i}\right\|}, o_{j}\right) \\
& =\frac{1}{N} \cdot \frac{\sum_{i} o_{i} \sum_{j} o_{j}}{\left\|\sum_{i} o_{i}\right\|}=\left\|\frac{1}{N} \cdot \sum_{i} o_{i}\right\|
\end{aligned}
$$

Indeed, the average offset will have a norm close to 1 if the offsets are higly similar, but close to 0 if they are anisotropic. We can now go further and link the MSM to the OCS.

$$
\begin{aligned}
\frac{1}{N^{2}}\left\|\sum_{i} o_{i}\right\|^{2} & =\frac{1}{N^{2}}\left(\sum_{i} o_{i}^{2}+\sum_{i} \sum_{j \neq i} o_{i} \cdot o_{j}\right) \\
& =\frac{1}{N}+\frac{N(N-1)}{N^{2}} \cdot \text { OCS } \\
\mathrm{MSM} & =\sqrt{\frac{1}{N}+\frac{(N-1)}{N} \cdot \text { OCS }}
\end{aligned}
$$

This result shows that the MSM is a strict (parabolic) improvement to the OCS, with a computable baseline decreasing with $N$. For BATS, $N=50$ (if all words are in the vocabulary), and thus the minimal MSM is $\sqrt{1 / 50} \approx 0.14$ even for anisotropic offsets (with an OCS $=0$ ). This score shows that offset concentration can be quickly amplified in unrealistic ways. Still, we can consider $d$ as a close representation of the offsets' relation. We display in Figure 7 the distribution of the similarities of the offsets to $d$.

\section{B Additional figures}




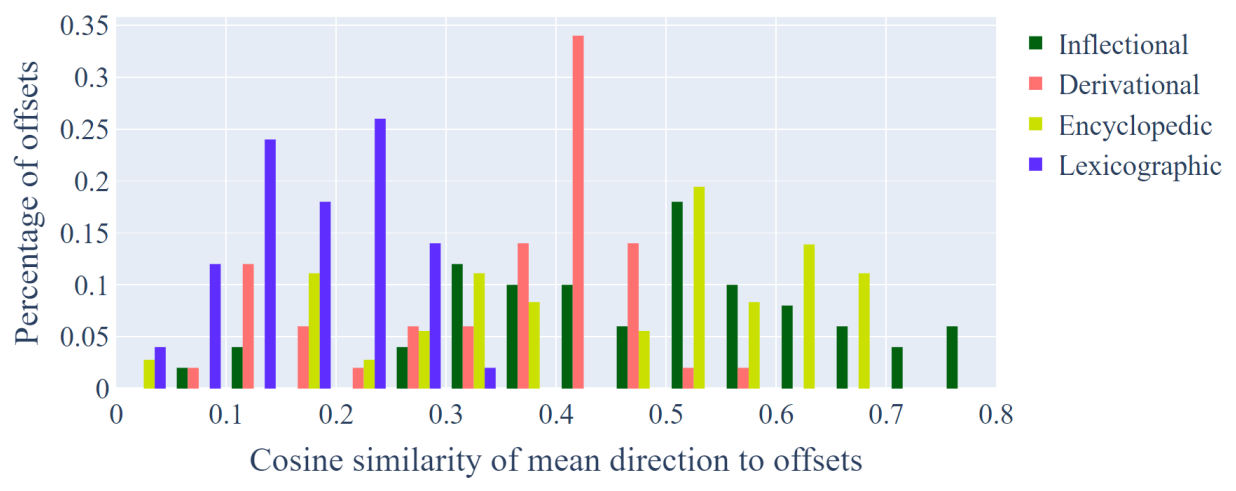

Figure 7: Distribution of the cosine similarity of vector offsets to the mean direction for the default word2vec embeddings for each category, then grouped by broad type. The mean similarity improves the similarity even for anisotropic offsets, as discussed. Variance changes seem category dependent.

\begin{tabular}{|c|c|c|c|c|c|c|c|c|c|c|c|c|}
\hline \multirow{3}{*}{ Embedding } & \multicolumn{3}{|c|}{ Inflectional } & \multicolumn{3}{|c|}{ Derivational } & \multicolumn{3}{|c|}{ Encyclopedic } & \multicolumn{3}{|c|}{ Lexicographic } \\
\hline & An & ogy & Metrics & An & $\log y$ & Metrics & & $\operatorname{ogy}$ & Metrics & & $\log y$ & Metrics \\
\hline & $\mathrm{N}$ & $\mathrm{H}$ & OCS PCS & $\mathrm{N}$ & $\mathrm{H}$ & OCS PCS & $\mathrm{N}$ & $\mathrm{H}$ & OCS PCS & $\mathrm{N}$ & $\mathrm{H}$ & OCS PCS \\
\hline wo & .680 & .099 & $\begin{array}{ll}.295 & .851\end{array}$ & .15 & .005 & $\begin{array}{ll}.156 & .679\end{array}$ & .198 & .20 & $\begin{array}{ll}.198 & .559\end{array}$ & .063 & .006 & $\begin{array}{ll}.031 & .539\end{array}$ \\
\hline Glo & .645 & .224 & $.345 \quad .860$ & .167 & .022 & $.237 \quad .710$ & .281 & .122 & . $255 \quad .623$ & .080 & .008 & $.004 \quad .550$ \\
\hline & 03 & .001 & .099 .700 & .12 & .00 & $.079 \quad .63$ & .162 & .0 & $.213 \quad .628$ & .0 & .00 & .024 \\
\hline & .360 & .226 & $\begin{array}{ll}.357 & .924\end{array}$ & .060 & .029 & $.224 \quad .805$ & .056 & .066 & $.251 \quad .674$ & .004 & .006 & $.034 \quad .552$ \\
\hline $\mathrm{BB}$ & .568 & .018 & $\begin{array}{ll}.217 & .821\end{array}$ & .288 & .229 & $.178 \quad .63$ & .041 & .07 & $\begin{array}{ll}.151 & .569\end{array}$ & .002 & .015 & $\begin{array}{ll}.016 & .517\end{array}$ \\
\hline GPT-2 t & .119 & .019 & .097 .651 & .359 & .248 & $.270 \quad .732$ & .014 & .050 & $.071 \quad .513$ & .003 & .011 & $\begin{array}{ll}.011 \quad .508\end{array}$ \\
\hline
\end{tabular}

Table 2: Comparison of analogy test scores and our OCS and PCS metrics for different word embeddings, grouped by broad type. $\mathrm{N}$ designates the Normal analogy test scores, and $\mathrm{H}$ the Honest analogy test scores where the input words are allowed to be predicted. OCS and PCS designate the Offsets Concentration Scores and Pairwise Consistency Scores. Bold means highest score for this type.

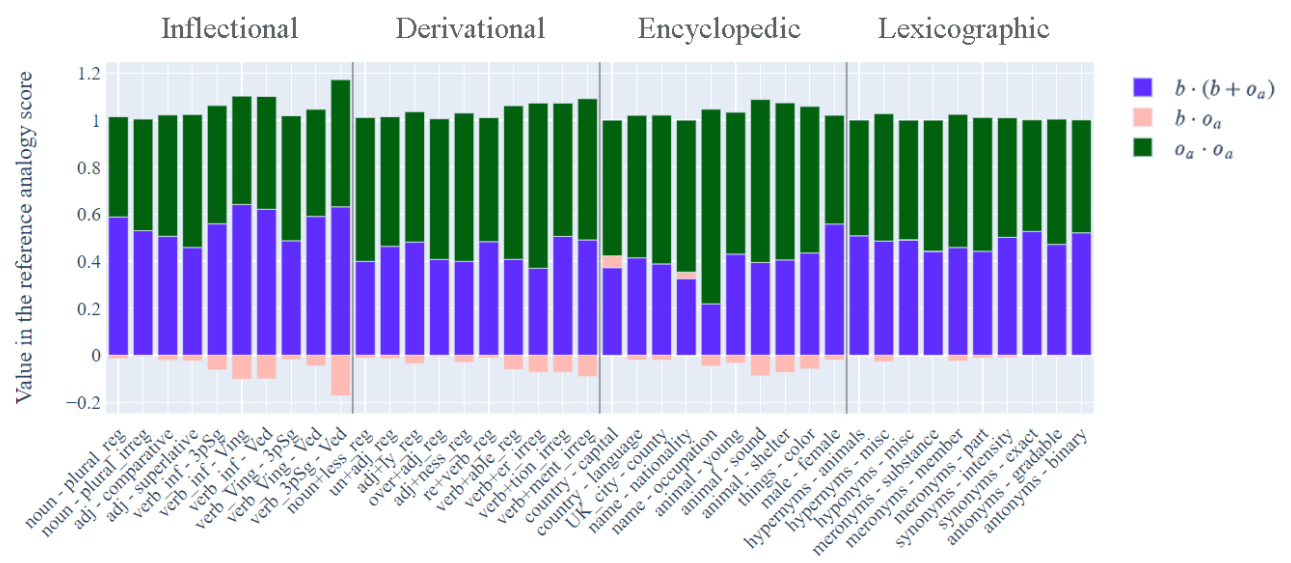

Figure 8: Decomposition of the analogy score if $o_{a}=o_{b}$ (the score being therefore equal to 1). The similar values of $b \cdot\left(b+o_{a}\right)$ and $o_{a} \cdot o_{a}$ indicates that the length of $o_{a}$ and $b$ is similar, and thus that the offset length does not explain the low value of $o_{a} \cdot o_{b}$ in the real decomposition. 